\title{
Technology as In-Between
}

\section{Stephen Read}

(C) Springer Science+Business Media B.V. 2011

\begin{abstract}
This commentary on Søren Riis's paper "Dwelling in-between walls" starts from a position of solidarity with its attempt to build a postphenomenological perspective on architecture and the built environment. It proposes however that a clearer view of a technological structure of experience may be obtained by finding technological-perceptual wholes that incorporate perceiver and perceived as well as the mediating apparatus. Parts and wholes may be formed as nested human-technological interiorities that have structured relations with what is outside - so that the outside constitutes an interiority in its turn which contextualises and situates the first. This nested structure raises questions about the way architects and urbanists see the built environment and understand inhabitation. It is hoped that this effort continues with conceptual and empirical work to research ways to make the human places of our built environment.
\end{abstract}

Keywords Architecture · Postphenomenology $\cdot$ Perception · Embodiment · Relationality · Space

We are required today to cross the boundaries of disciplines and to learn to speak with others of different expertise who don't share the same baggages of preconceptions. The complexity of the problems facing us demands this; the demand made all the more urgent in that it seems to be, at least in part, the splintering of areas of expertise that creates this complexity. But perhaps the greatest value of talking to others is the potential for the radical disruption of ideas that have become entrenched in disciplinary habit. I read this paper by Dr Riis (Riis 2011) as an architect by training and urban teacher and as a strictly amateur philosopher. I won't attempt here therefore to be as philosophically rigorous and systematic in reading it as Dr Riis has been in writing it: what I will try to do is convey what I see as its value as a challenge to ways of thinking in architecture and urbanism that may be past their sell-by dates. This review starts from a general solidarity with Dr Riis' project of building another

S. Read $(\varangle)$

Delft University of Technology, Delft, The Netherlands

e-mail: s.a.read@tudelft.nl 
view of the architecture and built environment we inhabit. I emphasise the word 'view' for reasons that will hopefully become apparent and because I have long held the opinion that we in the disciplines of the built environment have problems seeing the world from a perspective of the human and from the perspective of inhabitation.

I propose not to de-construct therefore, or add a point of view from an orthogonal position, but to try in the spirit of multidiscipinarity to talk in languages not well practiced - to begin to build what Bowker and Star call "boundary objects" (Bowker and Star 1999, 297) between different starting positions; points we can gather around to think further together. My wish here would be to see the beginnings of a constructive critique of architecture from a livedin human-centred perspective and of the construction of an alternative disciplinary view of the urban landscape. It is this possibility of taking on the contradictions of what Claude Raffestin calls the "geographical gaze" (Raffestin 2007) that I see as one of the most interesting contributions the postphenomenological perspective might offer. Phenomenology has had a considerable hand up to now in architecture and urbanism, but for me the real added value postphenomenology brings is in its relationality. In fact I don't think we will be talking here of the traditional phenomenological interests of subjectivity, architectural materiality or place, but rather of an alternative architectural and urban space to the ones we are already familiar with.

The postphenomenological view Dr Riis outlines does not frame experience through an interior mentality or subjectivity but takes experience out of the interior of the subject and, via technology, into the world of architecture itself. In so doing, it makes our technology of architecture an integral part of human situation and an integral part of a structure of experience embedded in the built environment itself. This in itself should alert architects and urbanists to sit up and take notice. The most surprising and valuable thing that first begins to emerge from Dr Riis' account is the idea that fields of perception may be shaped by technologies rather than simply by the geometries of optics. And rather than there being points of view on the world from simple singular places - or from universal and displaced points of view for that matter-there are many different and equally privileged technically supported 'optics' on the world (or on many worlds!).

We don't just use technologies to access an environment already known or directly perceived-we seem rather to become environed in the technologies themselves! The vague sense of subjection to the technology we feel in the London Underground: the sense of being a digit in an appropriately-termed mass-transportation system; of inhabiting a working diagram of the city with the gates and moving parts which engineer access to any specified place-all this suggests we are parts of a highly efficient system of conveyance, but also of an optic that defines and reveals the city as a world for us. At the same time there is something bounded and limited but complete in the way we see and do things through a system like this.

Dr Riis proposes in his paper a "new typology of architecture" founded in the way architecture frames experience. He builds on Don Ihde's conception of a postphenomenology and performs a commendable task in translating Ihde's ideas on our relationships to technology to architecture and producing a first view of what a postphenomenological architecture might look like. This opens up for me however, not just another new view on an old subject, but also the possibility of reconsidering and even replacing some longstanding and entrenched patterns of thought and practice in the disciplines of the built environment in general. Dr Riis' account ventures sometimes through doors and windows and beyond the architectural enclosure-opening, in my view, a way to how the nests we build may become not simply protective carapaces against a hostile outside, but also points of departure to wider domains and even home to the structured and filtered presence of wider (though not 'pervasive' or strictly 'global') worlds in the nest itself (see Read 
2009). When he does this there are hints to architecture and the urban as medium-but as medium, I propose, structured in a very specific and synthetic pattern that is both human and architectural. This pattern and the territorial and network structure it suggests raise serious questions about the way we think the architectural and urban and practice these disciplines today.

I begin therefore with an enthusiastic and somewhat premature reaction, linked to claims that have yet to be fulfilled. But I want to emphasise that this is a paper and an idea we can build on, and that the paper can be a worthy foundation to worthy work still to be done. The prospects that open up in taking on architecture and urban thinking through a simultaneously human and material-relational lens have been hinted at before: Hannah Arendt (1970) and Henri Lefebvre (2003) spring to mind; more recently, Peter Sloterdijk hints at a nested technorelational order of the environment as 'spheres' (Sloterdijk 2009). The work that is going to be needed to build an idea like this out further will be conceptual on the one hand; on the other we will need to get deep into the empirics, to map and understand this technorelationality in all its spatial and historical specificity, and begin to understand how we have constructed the worlds we inhabit, and how we need to construct it further to support practices and mediate experience in the future.

Dr Riis has structured his paper around four modes by which Don Ihde proposes technologies mediate experience. These modes depend for their particular characters crucially on boundary conditions set up in a human-technology-world schematic. So that embodiment, hermeneutic, alterity and background relations differ from each other in exactly where we bracket and frame relations within the total scheme and its parts. For example, the whole (human-technology-world) is parsed as (human-technology)-world in the embodiment relation. It is clear, at least to me, that one or two of these modes have a more central significance and more radical potential and deserve to be brought centre stage for the impact they may or should have on the ways we think about space. In particular we are confronted I believe with something really significant in the embodiment and background modes. These modes are 'transparent' and 'natural' on the one hand and 'atmospheric' on the other, and suggest the role of technologies as optics or scopes on the one hand and as a profound level of background organisation to our practices on the other.

Riis uses examples to explain these different modes in operation, but as the analysis develops it becomes clear there is a complex process going on in defining limited perceptual worlds and carefully bracketing particular technological objects in order to keep the elements of the analysis clearly differentiated. This is a useful exercise and may help in defining particular roles for architectural elements, but it takes our eye off another feature of technology in the human world - the fact that technologies seldom act alone, and that some may be foregrounded while others are backgrounded while all are in fact necessary for the activity or relation being described. While we use the toaster, what is the contribution of the bread bin, the counter, the kitchen, the electricity supply? When we remove the brackets it appears for a moment we live in a continuous 'atmosphere' of technology—so that picking out objects for analysis one by one is perhaps the best we can do. I suspect however there are both important continuities and discontinuities in all this that may give us clues about the ways we organise our human environment.

The architecture of the watchtower as a technology allows a clear view of the world-and the horizon of that view is presumably the line between earth and sky. But the real operational logic of the human-technology relation appears to be completed with the Panopticon, where the horizon is a scene integral with the technology and the world is the Panopticon. The Panopticon is, it seems to me, not so much more complex than the watchtower as more complete, embodying a world with its own operational logic and within which parts and 
wholes make sense in relation to one another. A certain engineered bounding, or "shielding", to use Nancy Cartwright's term (1999), sustains this world with its own internally embodied logic or reason. The question of boundaries here is not unlike the one that has exercised minds in thinking about scientific experimentation and measurement, because what is embodied is not just the object of the experiment but also the seeing, knowing and doing-including the apparatus and instruments that explicate and measure and the body that knows and does. The observer is part of the apparatus of experiment, needed there to complete the circuit of knowing and being known; the guard and the prisoner are parts of the Panopticon, needed there to complete the circuit of seeing and being seen.

The exact point at which vision is framed makes a difference to the way we understand technology and world. But only seeing the whole system will clarify the way the logics and intelligibilities of systems are set up and supported. In the case of measurement or observation in a scientific experiment, we may frame vision on the experimental object or instrument on the one side of a divide and bracket out an observer or measurer on the other. We may then imagine from the subject side that the object or measurement is part of the 'real' world or even of a universal frame of the laws of science or of a theory of everything. Understanding the role of the subject side in allocating significance to objects and measurements and in designing and maintaining apparatus gives a more complete picture of the logic of the result and how that logic is embodied in the whole set-up. In the architectural or urban environment we may frame an architectural object on the one side and viewers of that object on the other, and imagine the world in which we see the object is again the world seen in absolute space or even the universe. The world against which a house is seen is given however as part of a larger 'apparatus' we could call 'context' and in relation to which the house acquires its particular significance - in relation to which we see it in a particular way.

The inhabitant inside the house will on the other hand tend not to see or measure his or her home, and live in a whole embodied relation with it unless and until something intrudes from outside or something breaks down. There are questions about what 'world' is precisely in all of this, because to the inhabitant of a home making toast or doing the chores, home is a world structured familiarly around him or her, and outside is another world which may or may not intrude. We see this question arising in a very interesting way in the example of the apartment tower and in relation to the view from this interior world. Here, as we move higher in the block, what the inhabitant of an apartment sees through his or her window is not just a brighter or clearer or more extensive or less chaotic view: the view through the upper-floor window is framed in a different optic and in a world structured differently.

In fact what the view from above affords, as Riis rightly observes, is a pleasurable distance from the outside. Paradoxically, as more of the neighbourhood and the city become visible, what we experience from the upper-floor window is a retreat from the neighbourhood and the city. As we see more of it, it becomes more abstract and less immediate. What the pressing inside-ness of the surround of the apartment on the ground floor impresses on us on the other hand is the immediacy of the outside once the door is opened. This outside on the ground floor is not given to us as an overview or in abstraction, but as another surround of a public area or neighbourhood structured familiarly around us. Riis' analysis of the nest-like consubstantial interiorities of inhabitation can therefore begin to be developed outwards to the neighbourhood (or inwards to the bedroom) as the world opens to or closes on us in layered perceptual worlds. The consubstantiality of these worlds is no small matter as some of the answers to questions about the identities of modern or postmodern people may be wrapped up with these structured worlds and the ways they situate and involve people in real surroundings and ways of life. 
This structuring of worlds within worlds is something radically different to, but not necessarily less intelligible than (subject to no less viable an optic than) the overview from above. The example of the London Underground shows how our urban movement technologies are not only technologies of conveyance, but also optics on the very concrete places that they disclose and specify in their parts and wholes. Homes and neighbourhoods may operate without iconic diagrams but everyone knows the parts of their homes and neighbourhoods well enough to define home and neighbourhood and separate them clearly from each other. It is not simply that a door guards the transition between these two worlds; what marks them as nested together is the fact that the whole of one world is a part of the other! What is at stake here may be no less than 'cognitive' structure, or the structure of coherence, of the city or of the built environment in general. This structure has always tended to be overwhelmed in our geographical imaginings by the Euclidian 'objectivity' of the overview from abovethe view represented in the cartographic map. We in architecture and urbanism still carry around a default assumption that Euclidian distance matters-and some of us even believe still in a more or less exact spatial science that puts distance at its centre. The underlying principle here is that there is some kind of natural, preexisting, architectural or urban space to which we as creatures bound to geography are subject. What seems to me to be more significant about the built environment and its subjects however is the vast human organisation it incorporates; the way it is equipped and structured around our ways of life, with clearly felt discontinuities distinguishing different ways of life with differing qualities of publicness.

For me the real impact of Heidegger's analysis of Dasein strikes home with the way it makes us aware of the level of organisation in our human world. The ready-to-hand is more than just an attitude we adopt when wielding a hammer; it is the condition of the human world - not only saws and hammers are zuhanden, but monkeys and trees are as well (Harman 2010). And then we build zuhandenheit into the world around us-we technologise it. In our everyday dealings with the world we tend to bracket out the historical accumulation of this organisation, as well as the huge constructive effort that continues today, in much of the work we do, into maintaining and extending it, and coincidentally constructing nature as the background to this constructive effort. We maintain not just the order of the books in our libraries, and the filing in our schools and offices, but also the grades on our roads, the lighting and temperatures of rooms and the smooth invisibility of our floors, ceilings and lifts.

Another group in my profession, reacting to the physical deterministic excesses of the first, maintain a strong commitment to the absence of order, even the disorder, of our material built world. Order for them is instead constructed and maintained socially and subjectively, in a human and social world not altogether attached to the physical stuff. The fact is we are not strangers to our physical world; we are in it and profoundly part of it, and not only at home in our homes protected against a hostile outside; we are also at home in our neighbourhoods, in our cities, in our regions. And many of us these days are at home in cosy huddles that map as thin slivers of personal and family space, or academic, professional or cyberspace which span the globe. My profession in particular is involved in this home-making and we need a view on it that is both critical and constructive. According to Henk Oosterling, design, acutely aware of the possibilities for transparency and publicity, is likely to turn us into "viewers in a big open-air museum with no exit." Media and design render our environment transparent and impose a medium tempo which is that of our everyday life (Oosterling 2007). But while the Dutch landscape is being feverishly (and destructively) transformed into this media image, some of the facts on the ground testify to both the resilience and elliptical creativity of local cultures, capable of both absorbing and transforming this tempo in the lived-in in-betweens 
that constitute real architectures and places. Life happens not just at the centres of things (in transparent design-imaged environments) but also in the margins, separated from those centres not always by distance but by degrees of interiority or 'depth' from the most transparent of spaces.

The way we understand the possibilities and potentials of our architectural and urban practice depend to a large extent on what we understand our built environment to be-in this case on what its precise relation may be to human beings. Answers may come from an understanding of our relation to technology — as environment and as revealed through postphenomenology. Dr Riis' account has begun here to undermine some assumptions of mainstream accounts of geographical space. In particular, the subjective basis of human experience may be here subtly transformed into a material relation of the human subject with a layering of environments each more or less equipped and structured for particular ways of life. We need to understand these better in order to ally our efforts with the creativity of not only inhabitants but also of their places.

\section{References}

Arendt, H. (1970). The human condition. Chicago: Chicago University Press.

Bowker, G. C., \& Star, S. L. (1999). Categorical work and boundary infrastructures: Enriching theories of classification. In sorting things out. Cambridge, MA: MIT Press.

Cartwright, N. (1999). The dappled world: A study of the boundaries of science. Cambridge: Cambridge University Press.

Harman, G. (2010). Technology, objects and things in Heidegger. Cambridge Journal of Economics, 34(1), $17-25$.

Lefebvre, H. (2003). The urban revolution (R. Bononno, Ed. Trans.). Minneapolis: University of Minnesota Press.

Oosterling, H. (2007). Dasein as design, or: Must design save the world? (L. Martz, Trans.). Transcript of the Premsela Lecture 1st April 2007 given at the Koninklijk Instituut voor de Tropen, Amsterdam. Downloaded from http://www.premsela.org/_images/2007/01230.pdf.

Raffestin, C. (2007). Could Foucault have revolutionized geography? (G. Moore, Trans.). In J. W. Crampton \& S. Elden (Eds.) Space, Knowledge and Power: Foucault and Geography. Aldershot: Ashgate.

Read, S. (2009). Another form: From the 'informational' to the 'infrastructural' city. Footprint, 5, 5-22.

Riis, S, (2011). Dwelling in-between walls: The architectural surround. Foundations of Scinece 16(2-3), $285-301$.

Sloterdijk, P. (2009). Spheres theory: Talking to myself about the poetics of space. In Harvard Design Magazine 30. Cambridge, MA: Graduate School of Design, Harvard University.

\section{Author Biography}

Stephen Read is associate professor in the chair of Spatial Planning and Strategy in the Faculty of Architecture, Delft University of Technology. He completed his PhD in Delft and a fellowship at University College London before starting Spacelab Research Laboratory of the Contemporary City in 2002. He is interested in relationality in urban space and place and in the form and transformation of regions and cities. Besides being busy on a series of papers exploring Heidegger's space, he is busy with investigations of real urban places as socio-technical constructions, and is working on a book provisionally titled Urban Life and an edited volume on urban spaces as social technologies. 